\title{
Les nouvelles garanties des créances salariales
}

\section{Anna Filcheva}

\section{(2) OpenEdition}

\section{Journals}

Édition électronique

URL : https://journals.openedition.org/rdctss/2051

DOI : 10.4000/rdctss.2051

ISSN : 2262-9815

\section{Éditeur}

Centre de droit comparé du travail et de la sécurité sociale

\section{Édition imprimée}

Date de publication : 1 avril 2018

Pagination : 152-155

ISSN : 2117-4350

\section{Référence électronique}

Anna Filcheva, "Les nouvelles garanties des créances salariales », Revue de droit comparé du travail et de la sécurité sociale [En ligne], 1 | 2018, mis en ligne le 01 novembre 2021, consulté le 12 novembre 2021. URL : http://journals.openedition.org/rdctss/2051 ; DOI : https://doi.org/10.4000/rdctss.2051

\section{(c) (i) (9)}

Revue de droit comparé du travail et de la sécurité sociale est mise à disposition selon les termes de la Licence Creative Commons Attribution - Pas d'Utilisation Commerciale - Pas de Modification 4.0 International. 


\section{ANNA FILCHEVA}

Université de Plovdiv, "Paisi Hilendarski", Plovdiv

\section{LES NOUVELLES GARANTIES DES CRÉANCES SALARIALES}

Le 13 décembre 2017, l'Assemblée nationale a adopté la loi pour l'amendement et le supplément au Code du travail, promulguée et publiée au Journal officiel, n¹02 de 2017. La loi est entrée en vigueur le jour de sa promulgation, le 22 décembre 2017, à l'exception des dispositions octroyant un nouveau pouvoir de l'inspection du travail de déposer une demande d'ouverture d'une procédure d'insolvabilité de l'employeur entrant en vigueur le 31 mars 2018. Les dispositions finales de la loi apportent aussi des modifications à quatre autres lois parmi lesquelles le Code de procédure civile, la loi sur les créances garanties des travailleurs en cas d'insolvabilité de l'employeur et la loi sur le commerce. L'objectif de ces amendements et suppléments est d'introduire de nouvelles garanties de créances salariales à travers une augmentation des compétences de l'autorité de contrôle (I) et de la possibilité des salariés d'obtenir satisfaction de leurs créances salariales (II).

\section{I - L'ÉLARGISSEMENT DES COMPÉTENCES DE L'AUTORITÉ DE CONTRÔLE}

Les pouvoirs de l'inspection de travail ont été étendus dans trois directions. Il s'agit en premier lieu d'un élargissement des compétences de l'autorité de contrôle quand elle exerce le contrôle sur le respect du droit du travail $(A)$, en deuxième lieu de se pouvoirs supplémentaires dans le domaine du droit commercial - vérifier l'exactitude des faits concernant le paiement des créances salariales déclarés auprès de l'Agence d'enregistrement (B)- et en troisième lieu de déposer une demande d'ouverture d'une procédure d'insolvabilité de l'employeur (C).

\section{A - LES NOUVEAUX POUVOIRS DE L'INSPECTION DU TRAVAIL EN MATIÈRE DE CONTRÔLE DU DROIT DU TRAVAIL}

La modification de l'article 399, al.1 du Code du travail vient de reconnaître à l'inspection du travail le pouvoir d'exercer, outre le contrôle général du respect du droit du travail, celui du paiement des salaires et des autres créances salariales qui peut être exercé de manière autonome y compris lorsque la relation de travail est déjà résiliée. À cet égard, l'article 404, al. 1.12 nouveau du Code du travail permet à l'inspection du travail de mettre en demeure l'employeur de payer des salaires et d'autres créances salariales. L'inspection du travail peut être saisie par le salarié concerné ou agir d'office. Selon la disposition ajoutée à l'article 404, al. 3 du Code du travail, la demande du salarié ne peut être formulée qu'avant la saisine du juge. En cas de contradiction entre la mise en demeure de l'inspection du travail et la décision du tribunal, c'est celle-ci qui prévaut.

Avant, l'inspection du travail pouvait imposer une mise en demeure à l'employeur pour le paiement des créances salariales impayées, dans la mesure où le non-paiement 
en temps voulu de la rémunération constitue une violation de la législation du travail. La nouveauté réside dans le fait que cette mise en demeure peut être notifiée non seulement pendant la durée du contrat de travail mais aussi une fois la relation de travail résiliée. Ce changement s'inscrit en faux avec la jurisprudence constante de la Cour administrative suprême selon laquelle l'inspection du travail ne peut pas résoudre des litiges entre les salariés et les employeurs nés de relations de travail déjà résiliées ${ }^{1}$.

Dans le cadre de la nouvelle compétence de l'inspection du travail, il est également explicitement prévu un délai de paiement de certaines compensations dues à la rupture du contrat de travail, dont la compensation pour préavis non respecté, la compensation due par l'employeur lors de la résiliation unilatérale du contrat de travail dans certains cas prévus par le Code du travail et l'indemnité compensatrice de congés payés. Selon le nouvel al. 3 de l'art. 228 du Code du travail, le délai de paiement de ces compensations est fixé jusqu'à la fin du mois suivant la rupture de la relation de travail, sauf régime plus favorable prévu par convention collective. Ce changement ne profite pas aux salariés qui jusqu'ici pouvaient réclamer lesdites compensations à partir de la résiliation du contrat de travail.

\section{B - LES NOUVELLES COMPÉTENCES DE L'INSPECTION DU TRAVAIL DE VÉRIFICATION DE L'EXACTITUDE DU PAIEMENT DES CRÉANCES SALARIALES}

La Loi a également introduit des modifications de la loi sur le commerce. Selon l'art. 15, al. 4 et l'art. 129, al. 1 de la loi sur le commerce, les entreprises commerciales et les parts sociales ne peuvent être transférées que si les salaires, les compensations et les cotisations sociales des salariés, y compris ceux dont les contrats de travail ont été résiliés durant les trois années précédant le transfert, sont payés.

Avec les dernières modifications de la loi sur le commerce (entrée en vigueur le 16 février 2018), il a été établi que les associés/actionnaires qui vendent des entreprises commerciales et des parts sociales doivent déclarer le paiement des créances salariales auprès de l'Agence d'enregistrement qui en informe immédiatement l'inspection du travail. L'inspection du travail procède, sur la base d'une alerte ou de sa propre initiative, à une vérification des faits déclarés. Si elle constate une fausse déclaration, elle transmet les résultats de l'enquête à l'autorité de poursuite pénale, qui peut saisir le juge pénal bulgare.

\section{C - Les NouVEauX PouVOIRS DE L'INSPECTION DU TRAVAIL EN CAS DE PROCÉDURE D'INSOLVABILITÉ DE L'EMPLOYEUR}

La nouvelle disposition de l'art. 399, al. 3 du Code du travail, vient d'introduire le pouvoir de l'inspection du travail de demander au tribunal d'ouvrir une procédure d'insolvabilité de l'employeur. Deux conditions doivent être réunies selon l'article 625 de la loi sur le commerce :

- l'employeur doit avoir au moins deux mois de retard dans le paiement des créances salariales;

1 Décision $n^{\circ} 15296$ du 03.12.2012 de la Cour administrative suprême, section VI ; Décision $n^{\circ} 8519$ du 13.06.2012 de la Cour administrative suprême, division IV ; Décision nº 6583 du 15.03 .2015 de la Cour administrative suprême, section IV; 
- il s'agit des créances salariales exigibles pour rémunérations impayées concernant au moins un tiers des salariés ;

Concernant la deuxième condition, l'art. 245 du Code du travail doit être pris en compte qui précise que la loi garantit le paiement de $60 \%$ du salaire brut au salarié, et au moins le salaire minimum du pays, le restant étant exigible avec les intérêts légaux. L'effet de cette règle est qu'elle permet aux employeurs en difficulté financière de ne pas payer la totalité du salaire des salariés.

Le Médiateur de la République de Bulgarie, Maya Manolova, a fait un recours devant la Cour constitutionnelle demandant à ce que soit déclarée l'inconstitutionnalité de l'art. 245 du Code du travail, en raison de sa contrariété avec nombre de principes constitutionnels, y compris celui d'une rémunération correspondant au travail effectué. En désaccord sur ce point, la Cour constitutionnelle n'a pas atteint la majorité requise pour émettre un avis et a rejeté la demande du Médiateur².

La question principale qui se pose ici est de savoir si l'inspection du travail peut demander l'ouverture d'une procédure d'insolvabilité de l'employeur qui a, conformément à l'art. 245 du Code du travail, versé les salaires sur une période de plus de deux mois à au moins le tiers des salariés. Dans cette hypothèse, il existera également une créance salariale non satisfaite correspondant à l'écart entre le salaire versé ( $60 \%$ ou plus du salaire brut) et la rémunération convenue. Malheureusement, la réponse ne figure pas dans la loi. C'est pourquoi, on attend l'interprétation du juge.

\section{II - LA POSSIBILITÉ DES SALARIÉS D'OBTENIR SATISFACTION DE LEURS CRÉANCES SALARIALES}

La satisfaction des créances salariales est possible également à travers des poursuites individuelles selon une procédure désormais simplifiée (A). En cas d'insolvabilité de l'employeur, les salariés peuvent aussi bénéficier du fonds spécial qui garantit les créances salariales dans les conditions maintenant allégées (B).

\section{A - UNE PROCÉDURE SIMPLIFIÉE POUR DES POURSUITES INDIVIDUELLES}

Le nouvel alinéa 6 de l'art. 404 du Code du travail permet au salarié d'obtenir une copie de la mise en demeure délivrée par l'inspection du travail en vertu de l'art. 404, al. 1, p. 12 du Code du travail pour les créances salariales impayées par l'employeur. Il ne s'agit que des mises en demeure établies par l'inspection du travail dans le cadre de sa nouvelle compétence d'exercer un contrôle du paiement des créances salariales une fois la relation de travail résiliée.

Sur la base de la mise en demeure, le salarié peut saisir directement le tribunal civil en bénéficiant d'une procédure simplifiée pour poursuites individuelles prévue à l'art. 417 du Code de procédure civile, désormais complété avec un nouveau point 8. Contrairement à la procédure ordinaire, le juge vérifie si le document est dûment délivré et certifie une créance exécutoire contre l'employeur. L'employeur ne peut la contester qu'après la

2 Décision $n^{\circ} 1$ du 16 janvier 2018, promulguée au Journal officiel, nº 10 de 2018. 
délivrance du titre exécutoire. La poursuite devant le tribunal ne peut être effectuée qu'une fois la mise en demeure de l'inspection du travail devenue définitive, ce qui peut engendrer un certain retard.

\section{B - ÉLARGISSEMENT DES POSSIBILITÉS DE SATISFACTION EN CAS D'INSOLVABILITÉ DE L'EMPLOYEUR}

Suite à la réforme de l'art. 4, al. 1 de la Loi sur les créances garanties des travailleurs en cas d'insolvabilité de l'employeur, a été élargi le cercle des salariés qui peuvent réclamer leurs créances auprès du fonds spécial crée en vertu de la Loi et financé notamment par des cotisations sociales dues par l'employeur. Dorénavant, la Loi s'applique aux salariés qui sont ou qui ont été employés par un employeur impliqué dans une procédure d'insolvabilité, indépendamment de la durée du contrat de travail et de la durée du travail. II n'est plus nécessaire que la relation de travail soit en cours d'exécution à la date d'entrée en vigueur de l'ouverture de la procédure d'insolvabilité ou qu'elle ait été résiliée au cours des trois derniers mois précédant cette date.

La période de référence augmente également. L'art. 22, al. 1 de la Loi modifié prévoit que les créances salariales garanties sont les 6 derniers salaires mensuels et les indemnités dues calculées mais non versées par l'employeur au cours des 36 derniers mois calendaires précédant le mois au cours duquel la procédure d'insolvabilité a été inscrite auprès de l'Agence d'enregistrement.

Le montant mensuel maximum des créances salariales garanties continue d'être fixé annuellement par la loi sur le budget des assurances sociales de l'État et ne peut pas être inférieur à deux fois et demie le salaire minimum (510 BGN (260.76 EUR environ) pour 2018) à la date d'enregistrement de l'ouverture de la procédure d'insolvabilité auprès de l'Agence d'enregistrement. Le montant mensuel maximum des créances salariales garanties pour 2018 est de 1300 BGN (920.33 EUR environ). Selon le nouvel alinéa 3 de l'art. 22 de la Loi, quand il s'agit de compensations dues en vertu d'une loi ou d'une convention collective, les créances salariales garanties sont égales aux compensations impayées plafonnées à quatre fois le salaire minimum à la date de la rupture de la relation de travail.

La procédure commence à la demande du salarié qui dépose une déclaration à I'Institut national de sécurité sociale dans un délai désormais fixé à trois mois à partir de la date d'enregistrement de l'ouverture de la procédure d'insolvabilité ou, dans le cas des entreprises multinationales, à compter de la date à laquelle des salariés de l'employeur en Bulgarie ont été informés de l'ouverture d'une procédure d'insolvabilité dans un autre pays.

En conclusion, même si certaines modifications sont critiquables elles peuvent être évaluées positivement. II ne fait aucun doute qu'elles sont nécessaires en raison du grand nombre de retards de paiement des salaires. Elles garantissent aussi le droit constitutionnel à la rémunération prévu à l'art. 48, al. 5 de la Constitution bulgare. Le temps montrera si le but de ces réformes, d'assurer le paiement des salaires et d'autre créances salariales, a été atteint. 